\title{
What We Learn from the CTAD 2018 (Clinical Trials Alzheimer's Disease)
}

\author{
B. Vellas ${ }^{1}$, P. Aisen ${ }^{2}$, M. Weiner ${ }^{3}$, J. Touchon ${ }^{4}$

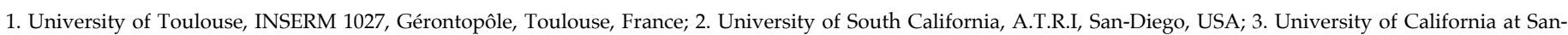 \\ Francisco, USA; 4. University of Montpellier School of Medicine, France
}

Corresponding Author: Bruno Vellas, University of Toulouse, INSERM 1027, Gérontopôle, Toulouse, France, vellas.b@chu-toulouse.fr

T Te are happy to publish the CTAD 2018 abstracts in the present JPAD issue. As you can see many new interesting studies are presented in this issue of the journal: from new drug trials to biomarkers, imaging studies, as well as new clinical outcomes. More specifically, we have several hot topics presentation on: 1. Major drug trials using bace inhibitors (verubecestat, lanabecestat, atabecestat, elenbecestat...) in the early phase of the disease (APECS early trials...). Both clinical, biomarkers (MRI, CSF, PET) and safety data will be presented. 2. New data on blood biomarkers including a keynote from R. Bateman, and presentations from Araclon and Roche biomarkers. 3. Results from phase III and IIB trials including a novel and multi-targeted oligosaccharide in patients with mild-moderate AD in China; the AMBAR (Alzheimer's Management By Albumin Replacement) study, the TOMMORROW trial: a trial to delay the onset of MCI due to AD and qualify a genetic biomarker algorithm, the 18-month STEADFAST trial of azeliragon in participants with mild Alzheimer's Disease; a longitudinal 148-week extension 4. Results 18 from F-AV-1451-A16: a clinicalpathological study of the correspondence between flortaucipir PET imaging and post-mortem assessment of tau pathology. 5. Latest developments in anti-amyloid monoclonal antibodies including aducanumab, and new results and data analyses of the BAN2401 study 201 in early AD. 6. New developments with safety and efficacy of lemborexant for sleep-wake regulation in patients with irregular sleep-wake rhythm disorders and Alzheimer's Disease dementia. 7. Advances with the ABBV-8E12, a humanized anti-tau monoclonal antibody, for the treatment of early Alzheimer's Disease. 8. Endpoints for early Alzheimer's Disease clinical trials: interpretation and application of the draft FDA guidance. And many others... It is important to underline that a not negligible number of abstracts concern non-amyloid targets (e.g; Tau-related targets but also targets outside the classical AD cascade).

We decided to allow quite long abstracts to give our readers the details they need to better understand each study. We can see that the field is moving toward blood biomarkers, tau PET imaging, combination therapies and e-cognitive outcomes. We are of course all concerned by the negative outcomes of some current drug trials, but as we can see with all these CTAD abstracts, the field is continually progressing, with some good perspectives: higher doses for anti-amyloid antibodies, early treatment, combination therapies and new biomarkers are likely keys to future success. Blood biomarkers and e-clinical trials may open a new era of large primary prevention trials. The CTAD Asia-China conference in Shanghai this past early September, gave us the opportunity to learn more about research in China, share with Chinese physicians ongoing work in Europe and the USA, and establish valuable contacts and collaborations.

The JPAD journal will contribute to the field. All JPAD issues are printed and shipped to the 1500 most important teams in the world including both academic and industry teams in the US, EU, Asia, and South America, all regions involved in AD clinical trials. The JPAD website reported 23,783 different users in the last 12 months. Moreover, 17 articles (ref 1-17) have been downloaded more than 1,000 times with a maximum of 14,341 for the CTAD abstracts (see ref). Springer-Nature is largely distributing the journal to thousands of medical libraries and schools of medicine worldwide. JPAD's Impact Factor will be released in 2019.

If we are able to learn from the failures in treatment trials and continue to work tirelessly, we will definitely be able to reach success for our patients and their families in the near future

\section{References}

1. CTAD: Symposia, Oral Communications, Posters, J Prev Alz Dis 2015;2(4):269396

2. Vellas B, Bateman R, Blennow K, et al. Endpoints for Pre-Dementia AD Trials: A Report from the EU/US/CTAD Task Force, J Prev Alz Dis 2015;2(2):128-135

3. CTAD: Symposia, Oral Communications, Posters, J Prev Alz Dis 2016;4:262379

4. Littlejohns TJ, Kos K, Henley WE, et al. Vitamin D and Dementia, J Prev Alz Dis 2016;3(1):43-52

5. Seifan A, Isaacson R, The Alzheimer's Prevention Clinic at Weill Cornell Medical College / New York - Presbyterian Hospital: Risk Stratification and Personalized Early Intervention, J Prev Alz Dis 2015;2(4):254-266

6. Downing AM, Yaari R, Ball DE, et al. Bridging the Gap between Research and Clinical Practice in Asymptomatic Alzheimer's Disease, J Prev Alz Dis 2016;3(1):30-42

7. Kahle-Wrobleski K, Andrews JS, Belger M, et al. Clinical and Economic Characteristics of Milestones along the Continuum of Alzheimer's Disease: Transforming Functional Scores into Levels of Dependence, J Prev Alz Dis 2015;2(2):115-120

8. Fotuhi M, Lubinski B, Trullinger M, et al. A Personalized 12-week "Brain 
Fitness Program" for Improving Cognitive Function and Increasing the Volume of Hippocampus in Elderly with Mild Cognitive Impairment, J Prev Alz Dis 2016;3(3):133-137

9. Hendrix S, Ellison N, Stanworth S, et al. Post Hoc Evidence for an Additive Effect of Memantine and Donepezil: Consistent Findings from DOMINO-AD Study and Memantine Clinical Trial Program, J Prev Alz Dis 2015;2(3):165-171

10. Cummings J, Fox N, Defining Disease Modifying Therapy for Alzheimer's Disease J Prev Alz Dis 2017;4(2):109-115

11. Wessels AM, Siemers ER, Yu P, et al. A Combined Measure of Cognition and Function for Clinical Trials: The Integrated Alzheimer's Disease Rating Scale (iADRS), J Prev Alz Dis 2015;2(4):227-241

12. Cummings J, Aisen P, Barton R, et al. Re-Engineering Alzheimer Clinical Trials: Global Alzheimer's Platform Network J Prev Alz Dis 2016;3(2):114-120

13. Anstey K.J, Eramudugolla R, Hosking DE, et al. Bridging the Translation Gap: From Dementia Risk Assessment to Advice on Risk Reduction, J Prev Alz Dis 2015;2(3):189-198
14. Tomaszewski S, Gauthier S, Wimo A, et al. Combination Therapy of AntiTau and Anti-Amyloid Drugs for Disease Modification in Early-stage Alzheimer's Disease: Socio-economic Considerations Modeled on Treatments for Tuberculosis, HIV / AIDS and Breast Cancer J Prev Alz Dis 2016;3(3):164172

15. Liu-Seifert H, Siemers E, Selzler K, et al. Correlation between Cognition and Function across the Spectrum of Alzheimer's Disease, J Prev Alz Dis 2016;3(3):138-144

16. Cebers G, Lejeune T, Attalla B, et al. Reversible and Species-Specific Depigmentation Effects of AZD3293, a BACE Inhibitor for the Treatmen of Alzheimer's Disease, Are Related to BACE2 Inhibition and Confined to Epidermis and Hair, J Prev Alz Dis 2016;3(4):202-218

17. Milne R, Bunnik E, Tromp K, et al. Ethical Issues in the Development of Readiness Cohorts in Alzheimer's Disease Researcn, J Prev Alz Dis 2017;4(2):125-131 